\title{
ELEMENTARY PROOF OF A NORM THEOREM
}

\section{HARLEY FLANDERS}

The result below was first obtained as a consequence of the theory of cyclic algebras [1]. ${ }^{1}$ The proof given here uses only the Hilbert norm theorem [2].

Let $K / k$ be a cyclic extension of degree $m n$ with generating automorphism $\sigma$. Let $K \supset F \supset k$ with $[K: F]=m,[F: k]=n$ so that $F$ is the fixed field of the subgroup $\left\langle\sigma^{n}\right\rangle$ of the cyclic group $\langle\sigma\rangle$. Let $a \in k$, $a \neq 0$, and $a^{m}=N_{K / k} A$. Then $a=N_{F / k} B$ for some $B \in F$.

To prove this, set $E=A A^{\sigma} \cdots A^{\sigma^{n-1}}$. Then $N_{K / F} E=\left(N_{K / F} A\right)$ $\cdot\left(N_{K / F} A\right)^{\sigma} \cdots\left(N_{K / F} A\right)^{\sigma^{n-1}}=N_{F / k} N_{K / F} A=a^{m}$, hence $N_{K / F}\left(a E^{-1}\right)$ $=1$. By the Hilbert norm theorem, $a E^{-1}=C / C^{\sigma^{n}}$ for a nonzero element $C$ of $K$. Hence $C^{\sigma^{n}}=a^{-1} E C$ and $C^{\sigma^{n+1}}=a^{-1} E^{\sigma} C^{\sigma}$. We set $B=A C / C^{\sigma}$ and have

$$
B^{\sigma^{n}}=\frac{A^{\sigma^{n}} C^{\sigma^{n}}}{C^{\sigma^{n+1}}}=\frac{A^{\sigma^{n}} E C}{E^{\sigma} C^{\sigma}}=\frac{A C}{C^{\sigma}}=B,
$$

hence $B \in F$. Finally,

$$
N_{F / k} B=A A^{\sigma} \cdots A^{\sigma^{n-1}} \frac{C C^{\sigma} \cdots C^{\sigma^{n-1}}}{C^{\sigma} C^{\sigma^{2}} \cdots C^{\sigma^{n}}}=E \frac{C}{C^{\sigma^{n}}}=a .
$$

Professor Jacobson pointed out to the author that the same argument can be used to derive Theorem 28 on page 47 of [3], starting from Theorem 27, which is a noncommutative form of the Hilbert norm theorem.

\section{REFERENCES}

1. A. A. Albert, Structure of algebras, Amer. Math. Soc. Colloquium Publications, vol. 24, 1939, Chap. 7, Theorem 16.

2. - Modern higher algebra, Chicago, 1937, Chap. 9, Theorem 5.

3. N. Jacobson, The theory of rings, New York, 1943.

California Institute of Technology

Received by the editors July 23, 1951.

${ }_{1}$ Numbers in brackets refer to the references at the end of the paper. 